\title{
Adaptação de material didático para u-learning: Sistema Odin
}

Title: Teaching Content adaptation for U-learning : Odin System

\author{
Marcelo Ricardo Quinta \\ INF - Instituto de Informática - UFG \\ Goiânia - GO \\ marceloquinta@inf.ufg.br
}

\author{
Fábio Nogueira de Lucena \\ INF - Instituto de Informática - UFG \\ Goiânia - GO \\ fabio@inf.ufg.br
}

\begin{abstract}
Resumo O aumento da popularidade do emprego de tecnologias móveis para acesso a internet viabiliza o uso de conteúdo educacional em qualquer lugar e instante. Contudo, usufruir desta flexibilidade exige esforço considerável, pois o responsável pelo conteúdo deve produzir uma quantidade de versões do material proporcional ao número de configurações dos contextos empregados. Este trabalho discute a adaptação de conteúdo nas ferramentas de ensino a distância atuais, os problemas associados ao emprego de diferentes dispositivos e situações e apresenta a solução Odin. O Odin oferece transparência na adaptação automática de áudio, vídeo, imagens e texto para uso em diferentes contextos, suporte ao SCORM e pode ser utilizado por aplicações já existentes.
\end{abstract}

Palavras-Chave: adaptação de conteúdo, acesso universal, mobilidade, u-learning

\begin{abstract}
The increasing of mobile technology usage popularity for internet access enables educational content access in anyplace, anytime. However, the use of this flexibility requires considerable effort, because the content sponsor must produce as many ones as the number of different devices configuration used. This work discusses the content adaptation support in actual learning management systems, the problems associated with the use of different devices to access educational content and presents Odin. The Odin offers transparency on automatic adaptation of audio, video image ant text for suitable adhibition of adapted content in different contexts, SCORM support and can be used as an extension by actual applications.
\end{abstract}

Keywords: content adaptation, universal access, mobility, u-learning 


\section{Introdução}

A internet é acessada cada vez mais por celulares, $t a$ blets, PDAs (Personal Digital Assistants) e aparelhos de TV Digital, dispositivos diferentes dos computadores convencionais. Isso fez o cenário do uso da informática mudar de "vários usuários por máquina" para "várias máquinas por usuário". E o educador pode aproveitar essas novas oportunidades, criando novas experiências de ensino.

Porém, ao mesmo tempo em que o uso desses dispositivos móveis na educação é inovador, ele traz obstáculos a serem vencidos pelos ambientes de ensino [1]. Cabe as plataformas a provisão de soluções para os problemas, fazendo com que os alunos tenham a oportunidade de utilizar os aparelhos, usufruir dos recursos existentes e ter experiências que tornem sua formação mais completa. Atualmente, tal suporte é restrito. Existem muitas funcionalidades ainda não cobertas pelos atuais sistemas de gestão de aprendizagem (LMS) [2], fazendo que tais softwares não dêem suporte ao u-learning, modalidade de ensino a distância que propõe a integração entre os pontos de acesso ao conhecimento e a situação (contexto) do usuário. O objetivo é o ensino "em qualquer hora, lugar e situação". Em tempo, contexto não é relacionado somente ao dispositivo que o interessado acessa o sistema. Ele pode englobar suas capacidades físicas, localização e qualquer outra informação que caracterize a situação que a pessoa se encontra.

Analisando os vários desafios que ainda devem ser enfrentados na área de $u$-learning, percebemos que um dos pontos importantes é o da facilitação da entrega de conteúdo adaptado para as diferentes aptidões, situações e hardware do discente, serviço definido como Acesso Universal [3]. Isso inclui a criação do objeto de aprendizagem (LO) propriamente dito e a correspondente personalização para cada meio empregado.

A criação de conteúdo já é parte do processo de trabalho do professor. Contudo, a personalização para vários contextos exige habilidade, conhecimento e esforço significativos. Cada aparelho ou situação possui peculiaridades que devem ser conhecidas e empregadas corretamente no processo de criação do LO adaptado. O professor pode consumir tempo valioso na adaptação e ainda corre o risco de produzir LOs de baixa qualidade.

O objetivo final deste trabalho é a definição e a construção de um protótipo que permita aos sistemas existentes para e-learning a abrangência de mais alunos, de forma apropriada e com eficiência, tanto para o aluno quanto para o professor. Isso é conseguido através da transparência na criação das versões personalizadas do material original (sem que o professor precise realizar a conversão) e na descoberta de contexto (sem que o aluno precise declarar explicitamente em que situação se encontra e qual versão do conteúdo ele deseja). Uma ilustração do cenário desejado é mostrada na Figura 1.

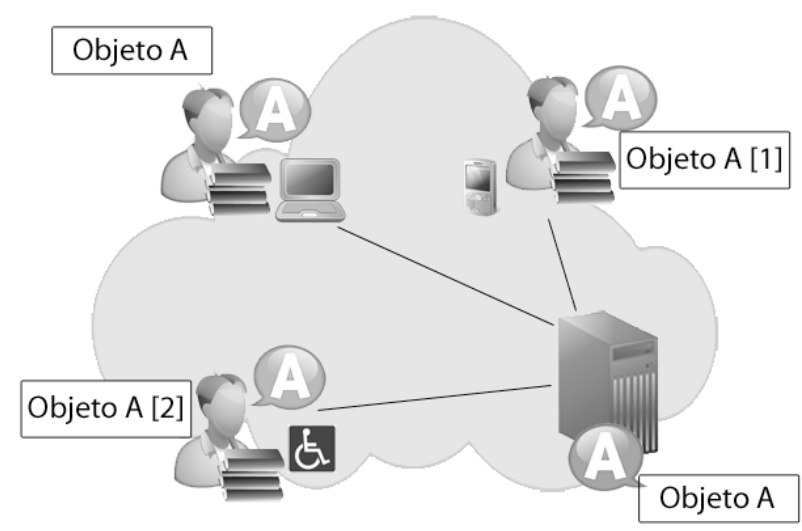

Figura 1: Estudantes em diferentes contextos acessando versões personalizadas de um recurso e assimilando o conteúdo.

\subsection{Método de trabalho}

O desenvolvimento deste trabalho foi dividido em cinco grupos de atividades:

- Levantamento bibliográfico Foram avaliados cerca de noventa artigos sobre $e$-learning, caracterização de sistemas para e-learning, análise de funcionalidades de sistemas para e-learning, m-learning, sistemas adaptados para $m$-learning, u-learning, sistemas para $u$-learning, tecnologias para adaptação de conteúdo, arquiteturas para sistemas móveis e ubíquos, conversão de arquivos e protocolos para descoberta de contexto. Tais referências bibliográficas foram obtidas através de consultas em portais de periódicos e conferências, e são utilizadas por todo o trabalho para embasar a argumentação.

- Análise de requisitos A definição do escopo, requisitos funcionais e não-funcionais, casos de uso e algumas restrições de projeto foram identificadas e registradas. Ao final dessa atividade, obteve-se um produto principal: a Especificação de Requisitos do Sistema Odin [4].

- Arquitetura de Software A elaboração da arquitetura de software do sistema partiu do momento posterior ao fechamento de escopo da aplicação, durante a análise de requisitos. Foram elaborados diagramas segundo as visões arquiteturais consi- 
deradas necessárias para o entendimento da aplicação, havendo muitos refinamentos durante todo o período de desenvolvimento do Odin. Nessa fase, foram escolhidas as tecnologias a serem utilizadas na implementação do protótipo. Ao final dessa atividade, obteve-se um produto principal: a Arquitetura de Software do Sistema Odin [5].

- Implementação do protótipo A especificação de requisitos e a arquitetura do sistema serviram de insumos para a construção do serviço que oferece todas as funcionalidades necessárias para a solução, com persistência de dados e acesso via internet. A implementação foi feita utilizando tecnologias Java, criando um produto que pode ser executado em diferentes sistemas operacionais.

- Testes do protótipo As funcionalidades foram testadas nos contextos alvo utilizando aparelhos, recriação de situações do aluno e emuladores.

\section{Desafios na produção de conteúdo para múltiplos contextos}

A disseminação de tecnologia tem facilitado e melhorado a criação de recursos para a aprendizagem. Porém, o mau planejamento do material pode causar desorientação e desmotivação do aluno, não permitindo que este possa acompanhar o curso e acessar a informação. Com isso, o engajamento do discente no processo de aprendizagem pode ser prejudicado. Tais resultados são polarizados dentro de ambiente móvel.

Quanto ao conteúdo educacional, muitas restrições devem ser tratadas nos diferentes contextos. Há muitos desafios e muitos deles similares aos existentes no $e$ learning tradicional. Outros estão relacionados ao modelo de ensino em si e a mobilidade. Podemos separá-los em duas principais categorias: pedagógicos e tecnológicos.

\subsection{Desafios pedagógicos}

Existem desafios para criação de atividades e conteúdos para $u$-learning que não estão ligados a tecnologia, mas ao modelo de ensino e a experiência do aluno e professor. Destes problemas, podemos citar a resistência a adoção de novas tecnologias e às novas práticas de aprendizagem, conhecimento da real situação do aluno, tempo aceitável de resposta reduzido e a disputa da atenção do conteúdo com outras fontes ao redor do discente.

\subsection{Desafios tecnológicos}

Os desafios tecnológicos do u-learning estão ligados principalmente a grande heterogeneidade de situações e dispositivos móveis, como a limitação do tamanho de tela e dispositivos de interação, quantidade heterogênea de cores suportadas, diferentes capacidades de processamento, memória e bateria, largura de banda e disponibilidade de planos de dados variáveis, rápida obsolescência dos aparelhos e falta de padronização no suporte as mídias pelos fabricantes de dispositivos. Para cada um deles existe uma solução ou contingência. Porém, não é conhecida uma estratégia que resolva todos ao mesmo tempo. Muitos deles estão inter-relacionados.

\section{Adaptação: uma possível solução}

Em um ambiente de $u$-learning, a experiência do usuário no sistema é fator importante na avaliação da qualidade do ensino da instituição. Logo, devemos tornar disponíveis os conteúdos para situações em que o usuário precise de atenção especial, seja por causa de seu aparelho ou condições excepcionais que ele se encontra. Como a maioria dos conteúdos é projetada para computadores de mesa, a alternativa para resolver a frustração de muitos usuários é a adaptação de conteúdo. "Mobilidade exige adaptabilidade " [6].

Adaptação é o processo de seleção, geração e modificação de conteúdo para adequação a diferentes ambientes computacionais e contextos de uso, o que pode ser feito também para serviços (chat, fórum, dentre outros). A técnica visa reconhecer e responder diferentes contextos de forma uniforme, não obstrutiva e se possível invisível, entregando ao usuário o que ele precisa, de forma personalizada. Para atingir esse objetivo, dois problemas devem ser resolvidos: consciência de contexto e conversão de conteúdo. Após conhecer o contexto e estabelecer uma abordagem para conversão do conteúdo, basta entregar ao usuário o recurso desejado.

Para entender o processo de adaptação, deve-se definir as estratégias de descoberta de contexto("Para"), local de adaptação ("Onde"), tipo de adaptação ("Quando"), seleção de conteúdo ("O quê") e a forma de conversão ("Como").

A descoberta do contexto permite que a aplicação possa saber para quem, onde e como está o usuário, com o objetivo de entregar conteúdo mais pertinente. Para isso, existem diversas abordagens, como a leitura de cabeçalhos HTTP [17], uso de parâmetros explícitos na requisição (do tipo CC/PP [19] ou UAProf [20]), ferramentas SMIL e o padrão MPEG-21 [18].

O local de adaptação refere-se ao agente que realizará a conversão. Conhecemos três opções: servidor (quem oferece o arquivo), cliente (quem recebe o arquivo) ou agente intermediário (proxy - localizado entre o cliente e servidor). Na abordagem do servidor, há a possibilidade 
de centralização e garantia de padronização das conversões. Porém, ele precisa de mecanismos para identificação do contexto e poder computacional suficiente para atender várias requisições de personalização ao mesmo tempo. A sobrecarga do cliente não existirá, pois este só consumirá o produto final já adaptado. $\mathrm{Na}$ abordagem de adaptação no cliente, o conteúdo original é entregue e a conversão ocorre diretamente no dispositivo do discente. Essa técnica pode demandar tecnologias e poder computacional no aparelho e não é garantida pela heterogeneidade de hardware. Finalmente, a adaptação via proxy ocorre em um agente intermediário separado do sistema (servidor), que só repassa as requisições do cliente. Dessa forma, tanto cliente como servidor não são sobrecarregados. O principal custo envolvido está na comunicação de rede entre os nós do sistema.

Os tipos de adaptação referem-se ao momento que a conversão do recurso ocorre. $\mathrm{Na}$ adaptação estática, o recurso é convertido antes que seja requisitado para o cliente, ou seja, o arquivo torna-se disponível antes de ser pedido. Com isso há o pré-processamento do recurso pelo servidor e maior alocação de espaço físico. Porém, ao receber a requisição, o arquivo já estará pronto para a resposta, que é retornada instantaneamente para o cliente. $\mathrm{Na}$ adaptação dinâmica, a conversão é feita durante o processo de requisição. Assim, logo que a requisição é feita, começa a adaptação do recurso, o que pode demandar mais tempo pelo processamento ocorrer em tempo de requisição, principalmente se não forem aplicadas técnicas de caching. Por outro lado, não há sobrecarga de espaço de gravação de adaptações. Os arquivos não ficam armazenados. Finalmente, a adaptação híbrida é o uso das duas estratégias anteriores combinadas, utilizando os prós de cada uma. Neste caso, a definição do tipo da adaptação que ocorrerá em cada nó deve seguir uma política préestabelecida.

Para a seleção de conteúdo para um contexto específico, é necessário saber se é realmente necessária a geração de uma nova versão do recurso. Em muitos casos, as restrições do aparelho exigem que conversões sejam realizadas. Em outros, versões já existentes podem ser utilizadas, evitando processamentos desnecessários e economizando espaço de gravação. Para resolver tal problema, temos duas abordagens: a trivial e a baseada em algoritmo de decisão. A abordagem trivial consiste na idéia de que, se existe versão diretamente ligada ao contexto, deve-se entregá-la. Caso contrário, realizar-se-á nova conversão. A abordagem tem a implementação simples, mas pode gerar desperdício de recursos para contextos análogos. Já a abordagem baseada em algoritmos de decisão é mais complexa e envolve problemas de decisão, geralmente solucionados por scoring e árvores de decisão. O material a ser entregue é comparado as versões já existentes.
Finalmente, caso seja necessário realizar uma conversão, há três formas de adaptação possíveis: escala, mudança de formato e mudança de modo. Na mudança de escala, há a alteração dos atributos do arquivo sem que haja mudança de formato. É indicada para os casos em que a alteração está relacionada a contextos em que a tecnologia já é suportada, mas certos atributos não são compatíveis (i.e. resolução de arquivo de vídeo). $\mathrm{Na}$ mudança de formato, supõe-se que a tecnologia não é suportada e, por isso, há a transformação de um recurso de uma codificação para outra. Finalmente, a mudança de modo, há a mudança do tipo do arquivo para outro ou uma coleção de outros (i.e. um vídeo transformado em uma síntese de um arquivo de som e várias imagens ou vice-versa), processo geralmente realizado por agentes humanos.

\section{Odin: adaptação e acesso ao conte- údo transparentes ao usuário}

O Odin é uma ferramenta para adaptação de conteúdo educacional para diferentes contextos. A solução funciona através da conversão de arquivos via agente humano ou por software no servidor. O objetivo é oferecer transparência tanto na conversão de arquivos para vários contextos, desonerando o professor, quanto no download de objetos educacionais personalizados para a realidade do aluno, tornando a experiência do $u$-learning agradável.

Existem três maneiras de uso dos dispositivos como ferramenta para o e-learning: acesso a mídia, comunicação ou ambas. O foco do Odin é tratar somente os casos de acesso a mídia (áudio, vídeo, imagens e texto). Questões sobre componentes de interface do usuário e ferramentas de colaboração não são tratadas.

Um ator na Engenharia de Software representa um papel executado por um usuário ou por um outro sistema interagindo com a solução modelada. Os atores que interagem com o Odin são:

- Administrador: Profissional com experiência em administrar sistemas de computação e com conhecimento em dispositivos móveis e contextos de ensino. Seu objetivo é gerenciar todas as configurações do sistema para garantir a qualidade do processo de conversão.

- Professor: Docente de um curso que oferece material de e-learning. Seu papel dentro do Odin é a inserção de recursos no repositório de LOs. Ele não possui responsabilidades relativas a administração do sistema. 
- Aluno: Discente cujo objetivo é consumir recursos de ensino online para suplementar o conteúdo das aulas. Pode não estar utilizando um computador comum e ter necessidades especiais.

- Conteudista: Profissional com experiência na edição de conteúdo. Seu objetivo no Odin é criar versões dos objetos de aprendizagem enviados pelo professor para os diferentes contextos homologados.

- Sistema LMS: Sistema de gerência de ensino em e-learning cujo interesse é utilizar o Odin como proxy de armazenamento/adaptação de conteúdo.

- Desenvolvedor: Mantenedor e desenvolvedor de aplicações cujo o objetivo é a implementação de conversores de mídia em conformidade com a API (Application Programming Interface) do Odin.

Os interessados no Odin observarão somente dois pacotes principais de componentes de prateleira: Interface gráfica e Web Services, ilustrados na Figura 02.

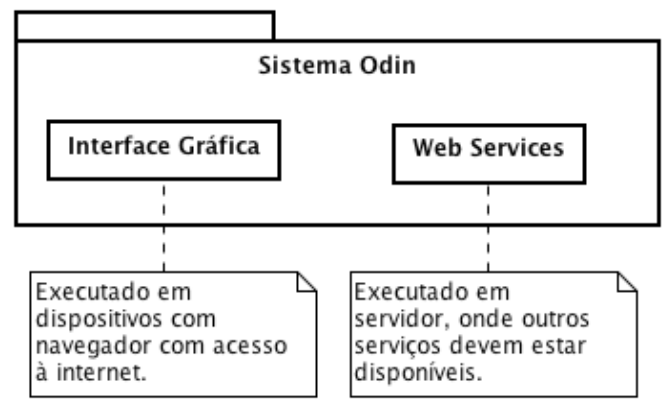

Figura 2: Visão de prateleira do Odin: as duas interfaces de acesso.

A interface gráfica é oferecida através de um endereço único na web (URL), onde o administrador tem acesso à manutenção do sistema, o conteudista possui acesso às funções relacionadas às suas tarefas de adaptação, o professor tem acesso ao serviço de upload de objetos de aprendizagem e os alunos têm acesso ao serviço de download de recursos personalizados. Já o componente de Web Services é responsável pela integração com outras aplicações que desejem fazer do Odin sua plataforma de adaptação de arquivos. Nesse caso, as requisições de alunos e professores são redirecionadas do LMS para o Odin, que atua como proxy de resolução de requisições. Para esse caso, não há interface gráfica, existindo somente uma descrição de mensagens HTTP que o cliente pode se comunicar, implementada no padrão REST [22].

Para o estabelecimento dos padrões de comunicação e adaptação do Odin, alguns princípios foram estabeleci- dos. São eles: transparência, experiência do aluno, flexibilidade e reusabilidade.

A transparência está na qualidade do serviço oferecido a professores e estudantes. Docentes não precisariam necessariamente conhecer as estratégias de edição do conteúdo para cobrir as necessidades especiais dos seus alunos. Eles devem somente construir o material, pensando nos pontos importantes a serem ensinados. Já os discentes não precisariam escolher a versão do recurso apropriado à sua situação. $\mathrm{O}$ aluno deve pedir o material e receber o conteúdo de forma personalizada.

A qualidade do contato de estudantes com o conteúdo é importante em uma ferramenta de $u$-learning. A experiência do aluno no Odin pode ser potencializada pela funcionalidade do recebimento do material otimizado para seu contexto.

A flexibilidade permite que um ambiente possa se adequar a uma instituição de ensino. O Odin é flexível ao permitir que novos conversores, inclusive humanos, sejam adicionados para fazer um trabalho aprimorado com os recursos enviados pelo professor. Além disso, a estratégia de entrega do material ("Somente original", "Somente adaptado" ou "Adaptado, senão original") também pode ser gerenciada, pois nem sempre um arquivo adaptado é atraente. $\mathrm{O}$ docente ainda pode ter o controle do conteúdo.

A reusabilidade faz com que a produtividade dos discentes aumente sem que precisem refazer trabalhos já realizados. O Odin oferece suporte ao padrão de objetos de aprendizagem SCORM [21], uma padronização de metadados que permite, dentre outros prós, uma catalogação dos dados mais eficiente. Logo, o Odin aumenta o potencial de reusabilidade dos objetos de aprendizagem, não somente por adaptar objetos de aprendizagem a outros locais, mas por facilitar sua localização e a classificação.

\subsection{Configuração do sistema}

O Odin oferece suporte a entrega de arquivos adaptados através da identificação do contexto em tempo de requisição e verificação de compatibilidade da realidade do usuário com as versões do recurso já existentes. O conteúdo é adaptado no servidor e antes da requisição. São oferecidas todas as formas de adaptação: escala, modo e tipo de mídia. Para que isso possa ser feito, algumas configurações do sistema devem ser realizadas pelo administrador através da interface gráfica do Odin:

- Contexto: Definição de um dispositivo, situação ou habilidade física do usuário. É composto por um identificador único e descrição. O Odin identifica o contexto de todas as requisições de downlo- 
$a d$. Por exemplo, toda vez que o Odin receber uma requisição de conteúdo vinda de um aparelho iPhone4S, um contexto chamado iPhone4S com descrição "Smartphone iPhone $4 S$ da Apple" será relacionado a requisição.

- Perfil: Contextos análogos são guardados em perfis. Gerenciados pelo administrador do sistema, eles contém nome, configurações de áudio, vídeo, imagens e texto compartilhados e a lista dos contextos relacionados. Por exemplo, o perfil "Dispositivos Apple" contém toda a configuração de mídia para contextos "iPhone 4S", "iPad" e "iPod touch".

- Configuração de mídia: Perfis contêm configurações de mídia para que, em tempo de adaptação, o conversor possa saber qual deve ser o formato do arquivo resultante. O Odin guarda informações de configurações de áudio, vídeo, imagem e texto. A configuração de imagem, por exemplo, é composta de largura, altura, resolução, codificação do arquivo (i.e. PNG, GIF) e presença ou ausência de zoom.

- Conversor: Dado um perfil e um tipo de mídia, o administrador deve escolher qual será o conversor responsável pela adaptação dos arquivos: os próprios conversores automáticos do sistema ou um usuário (conteudista) cadastrado. A cada requisição de adaptação cujo conversor seja humano, é gerada uma tarefa para que o profissional realize a criação da novas versões para os dispositivos homologados. Já os conversores computacionais são bibliotecas Java que realizam as operações estabelecidas na interface (API) do Odin. Novos conversores podem ser criados e adicionados na solução, acompanhando a evolução das mídias. A cada requisição de adaptação cujo conversor seja computacional, um novo processo é criado na máquina do Odin, convertendo os arquivos solicitados.

\subsection{Processo de envio e conversão}

O processo de gravação de um objeto de aprendizagem e a criação das versões adaptadas para os contextos inicia-se quando o professor posta o material. Isso pode ocorrer diretamente no Odin (Figura 3) ou via interface gráfica de uma aplicação LMS que usa o Odin como repositório, acessando-o via Web Services. Neste último caso, o sistema LMS redireciona os arquivos enviados pelo docente para o Odin, que responde ao sistema que o chamou. É importante destacar que o trabalho de criação do material por parte do professor encerra-se neste momento. Ele não precisa conhecer nenhum detalhe das conversões realizadas.

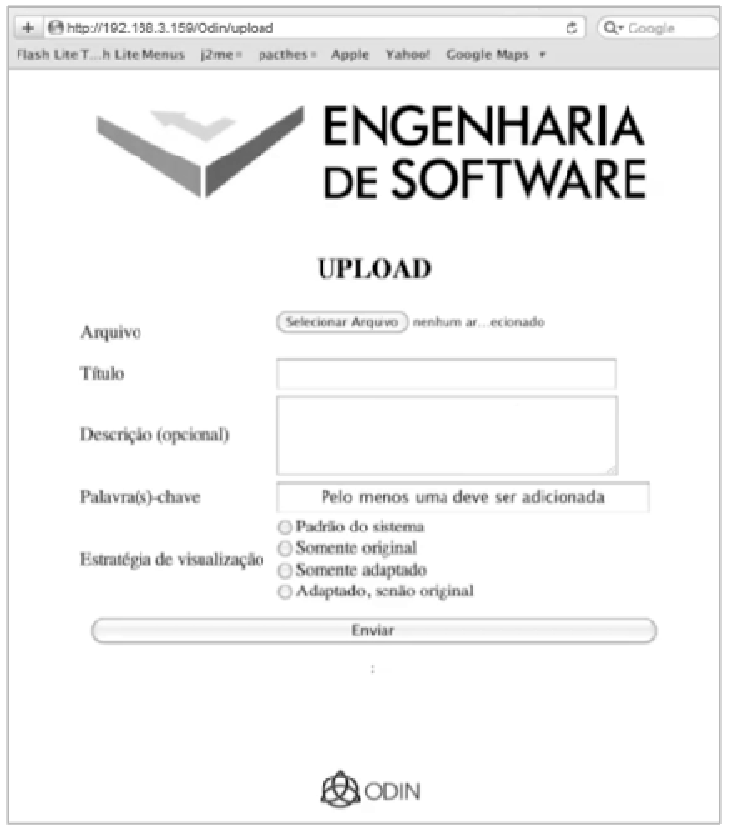

Figura 3: Tela de upload de objeto de aprendizagem.

Ao receber o objeto de aprendizagem, o Odin grava o original, registra as meta-informações (que podem estar também no descritor SCORM) e inicia o processo de conversão dos arquivos. As meta-informações serão utilizadas no futuro para indexação e pesquisa dos recursos educacionais.

Para cada arquivo do pacote enviado, com exceção do descritor SCORM, são criadas versões para os perfis existentes. Durante a adaptação para um perfil específico, verifica-se a existência de versão do mesmo recurso com as configurações de mídia (áudio, vídeo, imagens e texto) similares. Caso exista, é registrada a relação entre o arquivo e o perfil e a conversão não precisa ser feita ( $\mathrm{Na}$ Figura 4(a), observe que os perfis "Nokia" e "Applemobile" compartilham a mesma versão de recurso adaptado). Caso contrário, é requisitada uma conversão, feita à partir do conversor configurado para o perfil e o configuração de mídia. No caso do conversor do tipo software, é iniciado um processo no computador do servidor que faz a adaptação do arquivo em thread separada do sistema, pois essa pode ser uma tarefa onerosa em uso de CPU e memória. É importante destacar que o Odin realiza as chamadas dessas conversões através do uso do servidor web que permite a clusterização de máquinas para a realização do serviço. 
Um conversor do tipo software pode não estar disponível para o tipo da mídia e a configuração registrada para o perfil. Nesse caso, a adaptação será automaticamente relacionada ao conversor humano padrão cadastrado. Essa situação acontecerá em casos de contextos extremamente especiais. Por exemplo, para a conversão de uma imagem para um perfil relacionado a pessoas com deficiência visual, é necessário um trabalho mais específico, o que nem sempre é possível pelo conversor via software. Um conversor humano é considerado a melhor opção.

Após a adaptação, as versões dos arquivos são liberadas para download através do mesmo endereço eletrônico do arquivo original.

Finalmente, é importante destacar que o docente pode escolher se o conteúdo entregue será "Somente original" (Figura 3). Nesse caso, apesar de não ser indicado pelo sistema, o arquivo consumido pelos alunos será sempre o mesmo que o docente enviou, ou seja, não haverá criação de versão adaptada.

\subsection{Processo de download}

O Odin oferece funcionalidades de pesquisa de informações para objetos de aprendizagem registrados, tanto diretamente na ferramenta quanto por Web Service a ser utilizado em aplicações que o usam como proxy.

Após o objeto de aprendizagem ter suas versões criadas e disponibilizadas pelo sistema, basta que o aluno requisite o material diretamente pela interface gráfica ou por um LMS que usa a solução para que o Odin descubra o contexto do cliente e escolha a versão a ser entregue. $\mathrm{O}$ discente não precisa localizar a correta versão do objeto educacional. Ele só acessa a URL única que identifica o recurso. Um recurso pode ser um arquivo em específico ou todo o objeto de aprendizagem.

A descoberta de contexto pode ser realizada através do envio de identificador do contexto diretamente como parâmetro da requisição. Isso permite que contextos especiais, ligados à situação (e não ao dispositivo) possam ser criados. Tais parâmetros podem ser enviados por aplicações que desejem utilizar o Odin somente como um repositório para adaptação voltada a discentes especiais. Caso o identificador não seja enviado explicitamente, o sistema relaciona o contexto ao dispositivo utilizado. $\mathrm{O}$ software recupera o cabeçalho "User-Agent", presente nas requisições HTTP da internet, reconhecendo o aparelho com precisão através da biblioteca WURFL (disponível em http://wurfl.sourceforge.net/), atualizada periodicamente e que contém a maioria dos dispositivos móveis existentes. Dada a identificação do aparelho, o Odin verifica se este já foi registrado pelo sistema ou se as suas configurações são compatíveis com algum perfil existente. Isso é possível porque o WURFL oferece pesquisa de propriedades dos dispositivos. Caso o contexto não possa ser associado a nenhum perfil existente, ele é marcado como não suportado pelo sistema, mas isso não significa que o sistema retornará erro, pois a entrega do conteúdo depende da estratégia registrada pelo administrador ou professor.

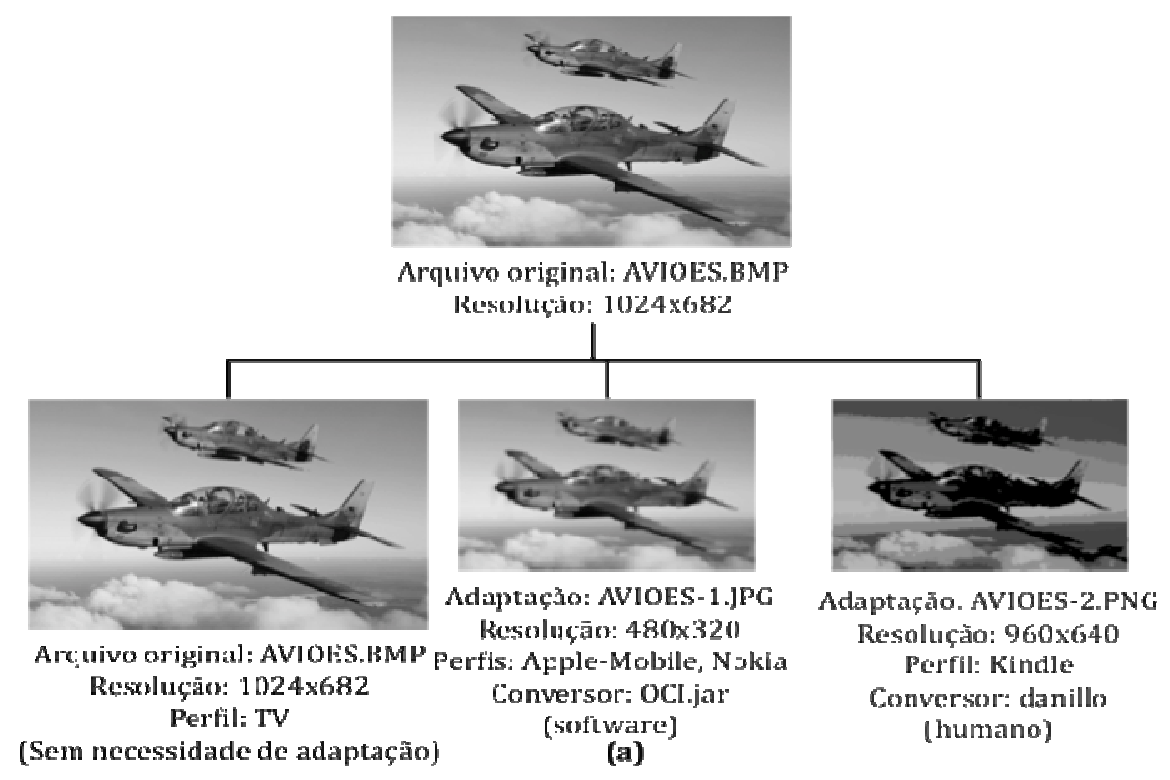

Figura 4: Exemplo de adaptação de um recurso de objeto de aprendizagem para diferentes perfis. Veja a diferença entre os recursos adaptados. 
Após a identificação, o Odin recupera os arquivos pertinentes ao perfil deste contexto e envia os arquivos ao cliente. Esta etapa depende da estratégia de entrega escolhida pelo professor e as configurações do sistema. Caso o professor tenha escolhido a opção "Somente original", é entregue somente a versão original. Caso o docente tenha escolhido a opção "Somente adaptado", a solução entregará o arquivo adaptado para o perfil associado ao contexto. Caso não exista arquivo relacionado ou o contexto não seja suportado, o sistema responde informações de erro seguindo os padrões da W3C (World Wide Web Consortium). Por fim, caso o professor tenha escolhido a opção "Adaptado, senão original", se o arquivo adaptado não existir ou não houver suporte para a realidade do usuário, será entregue o original.

\subsection{Implementação}

O protótipo do Odin foi criado para validar a arquitetura básica e dar suporte a um cenário de testes, onde alunos com seus dispositivos acessam o Odin, implantado em um servidor ligado à rede.

A Figura 5 mostra a arquitetura do Odin, composta de elementos divididos em interface, servidor e persistência. A interface gráfica foi implementada com JSP (Java Server Pages) e Servlets, que consomem os Web Services do sistema. O banco de dados utilizado foi o Apache Derby. Finalmente, o servidor foi desenvolvido com EJB (Enterprise Java Beans) para o atendimento às requisições e administração, Hibernate JPA(Java Persistence API) e HSQLDB (Hyper SQL Database) para a persistência, WURFL para identificação de contexto e JMS (Java Message Service) para a realização de conversões de conteúdo assíncronas via softwares adaptadores previamente cadastrados no Odin. Todas as tecnologias do servidor são executadas dentro do Glassfish, tecnologia open-source que oferece a clusterização das tarefas de adaptação e redundância do serviço.

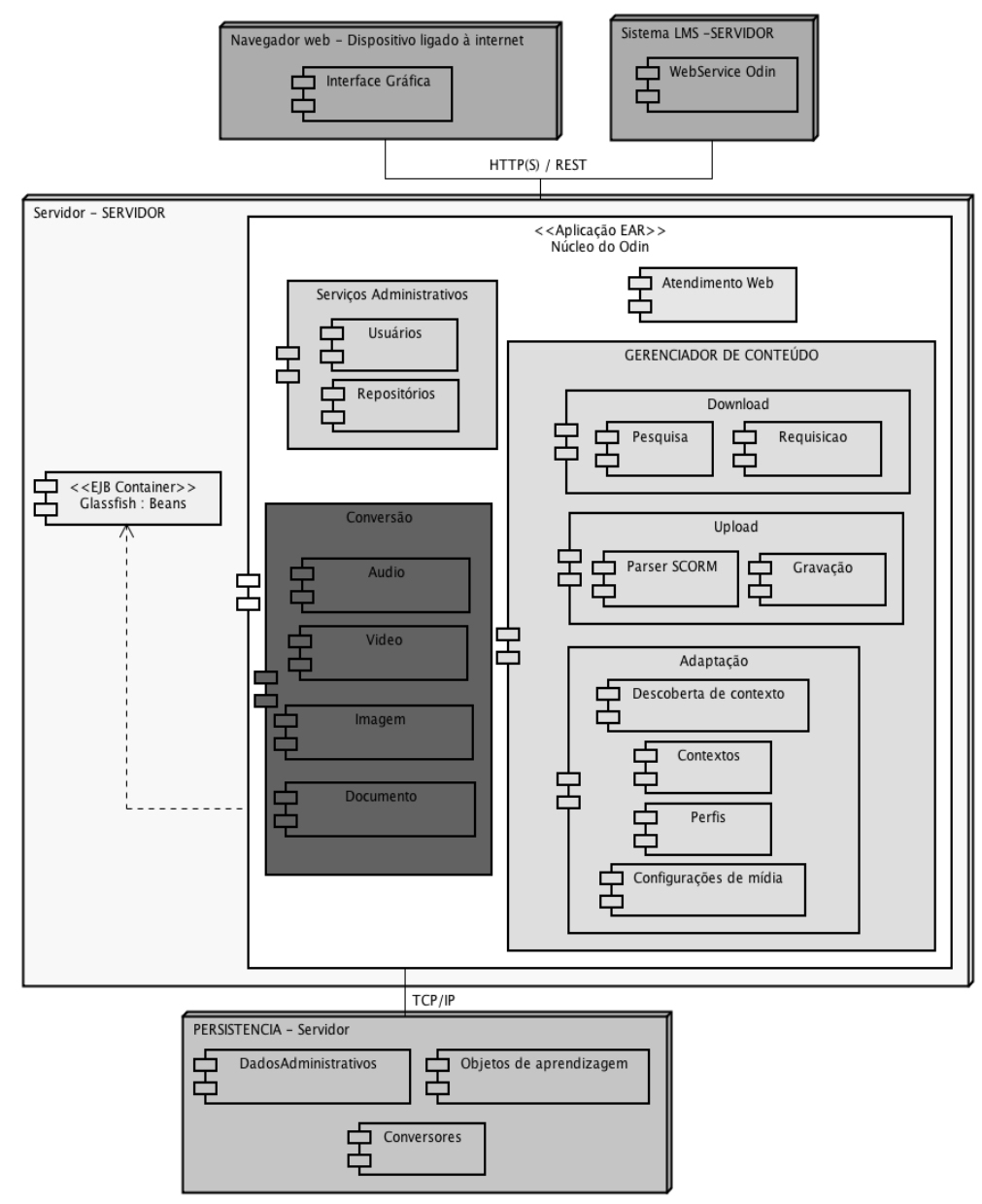


Figura 4: Arquitetura de implantação do Odin. São empregadas três estratos: cliente, servidor e persistência. Contudo, nada impede que servidores e bancos de dados sejam executados em um mesmo nó.

\subsection{Experimentação}

O teste simulado ocorreu com a participação de 12 voluntários, alunos de curso de graduação cursando o mesmo período, separados em dois grupos de 6 alunos. $\mathrm{O}$ Grupo 1 acessou o material por computadores de mesa e o Grupo 2 acessou por seus dispositivos móveis (desconhecidos) conectados à internet, com exceção de um aluno, deficiente visual, que utilizou seu computador.

Os dois principais questionamentos a serem respondidos foram:

1. O desempenho do uso dos recursos adaptados para o contexto do usuário é satisfatório?

2. O uso do sistema para acesso ao conteúdo foi satisfatório para os discentes?

O experimento aconteceu em 7 momentos: (1) configuração do sistema, (2) criação e envio do objeto de aprendizagem, (3) adaptação do conteúdo, (4) explicação de uso do sistema, (5) download e uso do conteúdo (6) realização de teste e questionário e (7) avaliação dos resultados.

A configuração do sistema consistiu na criação de três perfis: "Apple"(contendo as configurações de mídia e os contextos relacionados as configurações dos aparelhos iPhone e iPad), "Nokia" (contendo as configurações de mídia e os contextos relacionados as configurações dos aparelhos Nokia N95 e Nokia 5800) e "Deficiente visual" (relacionado ao contexto chamado "DV"). Foram relacionados os conversores de mídia padrão da aplicação para a conversão dos dois primeiros perfis e um conversor humano (conteudista) associado às adaptações do último perfil.

O objeto de aprendizagem utilizado para a aplicação foi relacionado a um conteúdo didático que os alunos estavam sendo introduzidos: "Tipo de dados Lista". O pacote incluiu uma imagem, um vídeo e um áudio. Ao final, o objeto foi inserido no sistema e foi marcada a estratégia "Adaptado, senão original".

Durante o processo de envio e conversão dos arquivos para os perfis relacionados aos fabricantes, não houve nenhuma intercorrência, sendo realizadas todas as adaptações. Para o arquivo de imagem, não foi necessária a criação de uma versão para cada perfil. A versão foi aproveitada entre perfis, ou seja, certos arquivos foram relacionados para mais de um perfil, economizando tempo de processamento e espaço de armazenamento. Para o perfil "DV", o conversor humano adaptou manualmente os recursos para a retirada de informações visuais, tor- nando-as textuais.

Divididos em dois grupos em salas diferentes, os alunos foram apresentados ao sistema e convidados a fazer consumo dos arquivos em dez minutos. É importante destacar que a identificação do contexto do aluno do perfil "DV" ocorreu através de leitura de informações explicitamente enviadas pelo requisitante (não visualmente pelo usuário, mas pelo sistema que este utilizou).

Após o tempo se esgotar, todos os sujeitos fizeram uma avaliação escrita com cinco questões objetivas sobre o conteúdo dado. O grupo que acessou o conteúdo pelos computadores de mesa obteve uma média 6,6 , enquanto o grupo que utilizou versões adaptadas dos recursos obteve a média 7,1 . Dada o pequeno espaço amostral somado a uma diferença de apenas meio ponto e ao ambiente controlado, não podemos tomar nenhuma decisão sobre a melhor opção para o ensino, mas podemos afirmar que os alunos conseguiram assimilar o conteúdo com o recurso adaptado. Não houve perdas.

Após a realização da prova, todos os alunos responderam a um questionário simples de percepção do conteúdo. As questões apresentadas estão na Tabela 1. Os seis discentes que utilizaram o sistema via computadores de mesa responderam apenas a primeira questão.

\begin{tabular}{l|l}
\hline$\#$ & Pergunta: "Qual é sua avaliação sobre..." \\
\hline 1 & . . a qualidade do material apresentado? \\
\hline 2 & $\begin{array}{l}\text { adaptado? } \\
\text { adactidade de consumo do material }\end{array}$
\end{tabular}

Tabela 1: Questionário realizado para os participantes do experimento.

Os resultados para a Questão 1 foram divididos pelos dois grupos para avaliar a qualidade do material adaptado em relação ao original. A comparação mostra que a adaptação foi satisfatória e bastante próxima do aluno em ambiente comum, havendo inclusive percepção mais positiva, com mais avaliações "Excelente". Porém, isso pode ter ocorrido simplesmente pelo fato do sucesso no consumo do conteúdo, visto que normalmente a visualização de recursos falha para novos contextos. 


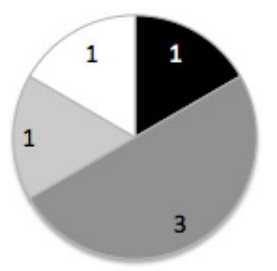

Grupo 1

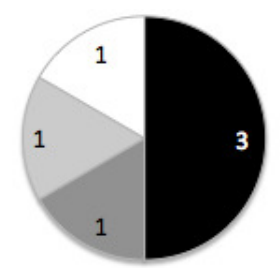

Grupo 2
Figura 5: Respostas para a Questão 1.

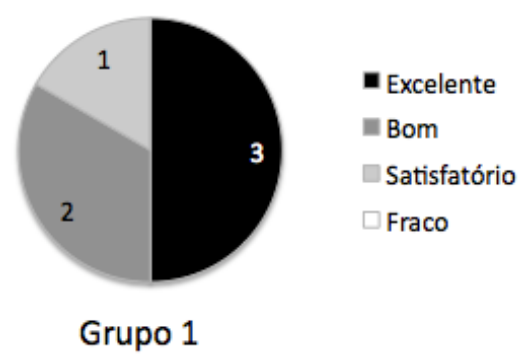

Figura 6: Respostas para a Questão 2.

Outro aspecto analisado foi a facilidade para o consumo do material adaptado. Na Figura 6, os resultados mostraram que os estudantes tiveram facilidade no consumo do conteúdo, inclusive o sujeito com deficiência visual. Este elogiou a estratégia do conteudista, que fez a transcrição da imagem em texto, uma das formas de promover a acessibilidade, visto que o computador deste indivíduo tinha uma aplicação leitora de texto da tela. Além disso, podemos citar que a indexação única do recurso para o estudante promoveu o acesso transparente ao discente, o que facilita o uso do sistema.

Finalmente, é importante destacar que houve sucesso na entrega de conteúdo para todos os dispositivos utilizados durante o experimento, mesmo que suas configurações não fossem conhecidas antes do download. Isso ocorreu porque os dispositivos dos usuários eram compatíveis com os perfis homologados: três aparelhos com tecnologia Android empregados pelos alunos foram compatíveis com o perfil "Apple".

\subsection{Trabalhos relacionados}

A adaptação de conteúdo é uma das áreas da computação sensível ao contexto. Uma das suas principais aplicações está na educação, alvo deste trabalho.

Dos trabalhos internacionais, podemos destacar o recente trabalho de Zhao [7], que apresentou um ambiente de adaptação em contextos com muitas variáveis. Além deste, Reveiu [8] apresentou uma arquitetura projetada para adaptação de conteúdo multimídia.

Dentre os trabalhos brasileiros, podemos citar Bartholo [9] e Barbosa [10]. O primeiro estabeleceu elementos e diretrizes para apoiar a adaptação de ambientes virtuais de aprendizagem para dispositivos móveis. O segundo apresentou um estudo sobre aplicação da computação móvel e ubíqua no contexto de um curso de graduação. $\mathrm{O}$ projeto Amadeus [11] também oferece adaptação de conteúdo via dispositivo móvel.

\section{Considerações finais}

\subsection{Conclusão}

O Odin é uma ferramenta para adaptação de conteúdo educacional para diferentes contextos. A solução funciona através da conversão prévia de arquivos via agente humano ou por software no servidor e é oferecida como um serviço a ser utilizado pelas plataformas de ensino existentes, como um serviço de base de dados de objetos educacionais. Seu objetivo é oferecer transparência tanto na conversão de arquivos para vários contextos, desonerando o professor, quanto no download de objetos educacionais personalizados para a realidade do aluno, tornando a experiência do u-learning agradável.

Esse trabalho discutiu alguns dos problemas relacionados com a adaptação automática de conteúdo para vários contextos. Os problemas vão desde os pedagógicos até tecnológicos e existem propostas para tratar cada um deles isoladamente. Contudo, tais dificuldades ainda não foram cobertas pelas atuais soluções de gestão de ensino e a combinação delas é inviável de ser trabalhada pelo professor.

Com o objetivo de viabilizar o e-learning, o Odin auxilia professores na recriação de conteúdo adaptado para cada contexto, no qual é planejado o suporte sem que os docentes tenham que se preocupar com as dificuldades do processo. Além disso, a ferramenta torna a operação de visualização de arquivos menos frustrante (maior qualidade), já que o material é automaticamente personalizado para o contexto utilizado.

Como diferenciais tecnológicos do sistema, provenientes de requisitos levantados na pesquisa, podemos destacar que:

- Nem todos os problemas de adaptação podem ser resolvidos pelo computador. Assim, o Odin oferece flexibilidade quanto aos conversores (agente humano ou computacional), o que viabiliza a configuração de conversões apropriadas para cada contexto.

- As soluções de gestão de ensino dão pouco suporte a adaptação de conteúdo. Devido a isto, existe a possibilidade no Odin de incorporação a qualquer sistema de gerenciamento de ensino, atributo o 
qual não foi encontrado em outras soluções pesquisadas.

- Atualmente, não existem versões da maioria dos objetos de aprendizagem para novos contextos. $\mathrm{O}$ Odin contempla o conteúdo existente. Dessa forma, ele abre a perspectiva de reutilização da enorme base de objetos educacionais disponíveis para uso em contextos diferentes daqueles para os quais foram criados. Assim, bases atuais podem empregar o Odin para atingir públicos que fazem uso de outros cenários não contemplados inicialmente.

\subsection{Resultados e contribuições}

Além da implementação de referência, da documentação de requisitos e da arquitetura de um sistema de adaptação de conteúdo, este trabalho incluiu um estudo detalhado sobre a cobertura de funcionalidades das soluções LMS existentes. Com isso, também verificamos a necessidade de suporte ao m-learning e $t$-learning por parte das aplicações, não só na adaptação, mas na disponibilização de muitas ferramentas de ensino.

O projeto Odin também gerou contribuições indiretas, em formato de software, relacionadas a projetos finais de cursos de alunos de Ciência da Computação da Universidade Federal de Goiás. São eles:

- OCA: Otimizador de Conversão de Áudio API de conversão de áudio para criação facilitada de programas de geração de arquivos de áudio adaptáveis para diferentes contextos, incluindo o da TV Digital Brasileira [12].

- OCV: Otimizador de Conversão de Vídeos API de conversão de vídeo para criação facilitada de programas de geração de arquivos de vídeo adaptáveis para diferentes contextos [13].

- OCI: Otimizador de Conversão de Imagens API de conversão de imagens para diferentes formatos, usando parâmetros como cor, tamanho e extensão [14].

- Interface móvel do Player Odin utilizando JavaFX Projeto e protótipo de interação de um software a ser executado em dispositivos móveis para acesso a funcionalidades de um sistema LMS [15].

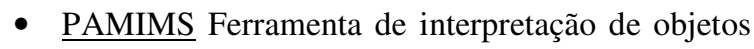
de aprendizagem no padrão IMS-QTI, que executa avaliações em aparelhos com mecanismo de inte- ração reduzidos, como celulares e controles de televisor. Ao final, foi produzido software que não só executava tais testes, mas também os enviava a resposta para servidor da instituição e gerava um relatório para o professor [16].

\subsection{Trabalhos futuros}

Melhorias em relação aos serviços oferecidos ao professor e às soluções LMS e a realização de novos testes são atividades imediatas com o propósito de tornar a versão corrente do Odin mais próxima de um emprego real, por vários professores e, dessa forma, receber retroalimentação que oriente trabalhos posteriores.

Para o professor, deve ser feita a criação de uma interface de gerência de seus objetos de aprendizagem, onde ele aprova ou reprova as adaptações feitas pelo sistema, além de poder escolher exatamente qual arquivo ele deseja adaptar. Atualmente, o Odin adapta todo o material enviado.

Para as soluções LMS, deve ser feita a implementação dos serviços de configuração do Odin via Web Services. Dessa forma, mantenedores dessas soluções poderão embutir o Odin diretamente dentro de suas aplicações, tornando seu uso facilitado. Atualmente, é necessário que o administrador mantenha as configurações pela interface gráfica do Odin.

Em relação aos testes, há o planejamento da implementação da integração com o Moodle (Disponível em http://moodle.org/), um sistema de gerenciamento de ensino bastante utilizado no mundo.

\section{Referências}

[1] L. R. de Oliveira, R. D. Medina, Desenvolvimento de objetos de aprendizagem para dispositivos móveis: uma nova abordagem que contribui para a educação. Revista Novas Tecnologias na Educação, v. 5, págs. 9-18, 2007.

[2] M. R. Quinta, F. N. de Lucena, An evaluation of e-learning plataforms funcionalities and the problem of m-learning and t-learning support. In Proceedings of the 7th International Conference on Information Systems and Technology Management, São Paulo, 2010.

[3] S. J. H. Yang, I. Y. L. Chen, Universal access and content adaptation in mobile learning. In Proceedings of the sixth international conference on advanced learning technologies, Kekrade, págs. 1172-1173, 2006. 
[4] M. R. Quinta, F. N. de Lucena, Odin: Viabilizando e-learning em múltiplos dispositivos. In Anais do XVI Workshop de Informática na Escola. Belo Horizonte, págs. 1397-1400, 2010.

[5] M. R. Quinta, F. N. de Lucena, Problemas e soluções em m-learning e a adaptação de conteúdo de objetos de aprendizagem para diferentes dispositivos. In Anais do XXI Simpósio Brasileiro de Informática na Escola. João Pessoa, 2010.

[6] M. Satynarayanan, Fundamental challenges in mobile computing. In Proceedings of the 15th ACM Symposium on principles of distributed computing, Philadelphia, págs. 1-7, 1996.

[7] X. Zhao, X. Wan, T. Okamoto, Adaptative content delivery in ubiquitous learning environment. In Proceedings of the sixth international conference on wireless, mobile and ubiquitous technologies in education, Kaohsiung, págs. 19-26, 2010 .

[8] A. Reveiu, I. Smeureanu, M. Dardala, Content adaptation in mobile multimedia system for $\mathrm{m}$ learning. In Proceedings of the 7th international conference on mobile business, Barcelona, págs. 305-313, 2008.

[9] V. de F. Bartholo , M. A. Amaral, M. I. Cagnin, M-ava: Modelo de adaptabilidade para ambientes virtuais móveis de aprendizagem. In Anais do XX Simpósio Brasileiro de Informática na Educação. Florianópolis, 2009.

[10] J. Barbosa, R. Hahn, S. Rabello, S. C. C. S. Pinto, D. N. F. Barbosa, Computação Móvel e Ubíqua no Contexto de uma Graduação de Referência. Revista Brasileira de Informática na Educação, 15(3):53-65, 2007.

[11] L. L. Lobato, B. de S. Monteiro, A. S. Gomes, Amadeus-MM: LMS com integração de serviços multimídia. In Anais do XX Simpósio Brasileiro de Informática na Educação. Florianópolis, 2009.

[12] O. F. C. Filho, OCA: Otimizador de Conversão de Áudio. Trabalho de Conclusão de Curso, Universidade Federal de Goiás, Novembro 2009.

[13] F. M. Lobo, OCV: Otimizador de de Conversão de Vídeos, Trabalho de Conclusão de Curso, Universidade Federal de Goiás, Novembro 2009.

[14] J. O. Barbosa, OCI: Otimizador de Conversão de Imagens, Trabalho de Conclusão de Curso, Uni- versidade Federal de Goiás, Novembro 2009.

[15] L.C. Alves, Interface móvel do player Odin utilizando JavaFX, Trabalho de Conclusão de Curso, Universidade Federal de Goiás, Novembro 2009.

[16] L. H. M. Ribeiro, Geração automática de avaliações para a web: Plugin para avaliações usando o padrão IMS-QTI. Trabalho de Conclusão de Curso, Universidade Federal de Goiás, Novembro 2009.

[17] T. Lemlouma, N. Layaida, Adapted content delivery for different contexts. In: Proceedings of the 2003 Symposium on applications and the Internet, Sophia Antipolis, págs. 190-197, 2003.

[18] MPEG-21, http://mpeg.chiariglione.org/standards/mpeg21/mpeg-21.htm, Março de 2012.

[19] World Wide Web Consortium, CC/PP Information Page, http://www.w3.org/Mobile/CCPP/, Março de 2012.

[20] World Wide Web Consortium, UAProf, UserAgent, Mobile, http://www.uaprof.com/, Março de 2012.

[21] Advanced Distributed Learning, SCORM Overview. http://www.ostyn.com/standards/docs/ scorm2004overview.htm, Último acesso em Março de 2012.

[22] R. T Fielding. Architectural styles and the design of network-based software architectures. Tese de Doutorado. Universidade da Califórnia. 2000. 